strongly shielded-electronic transition (shown in absorption, in fluorescence, and in optical sensitizing) proper to these ensembles is polarized in a direction perpendicular to the plane of the component molecular ions, that is, parallel to the axis of the packet or micelle-and, therefore, to a 110 plane of the crystal. The coupling of this with the azimuthal component of the electronic energy of a filled zone could proceed, therefore, as for an adsorbed molecule. In this connexion, it is significant that we find that non-planar cyanine dyes do not aggregate in the nematic state, while their planar isomers do so.

The considerations advanced are equally applicable to acid sensitizing dyes, for example, erythrosin, etc., of the xanthene type. These also are adsorbed edgewise and orthogonally to an octahedral surface, though of silver ions ${ }^{2}$. The molecules of these dyes are also planar, and $a$ fortiori, because having a fused polycyclic skeleton.

Research Laboratories,

Eastman Kodak Company,

S. E. SHEPPARD.

R. H. LAMBERT.

Rochester, New York. April 25.

1 NATURE, 145, 386 (1940).

2 Sheppard, S. E., Lambert, R. H., and Walker, R. D., J. Chem. Phys., 7, 265, 426 (1939).

s Sheppard, S. E., Vanselow, W., and Happ, G. P., J. Phys. Chem., $44,411(1940)$

- Kameyama, N., and Fukumoto, T., J. Soc. Chem. Ind. (Japan), 42, 489 (1939).

5 von Hippel, A., Z. Phys., 67, 707 ; 68, 309 (1931); 75, 145 (1932).

- Eggert, J., and Biltz, M., Trans. Farad. Soc., 34, 892 (1938).

7 Jelley, E. E., NATURE, 139, 631 (1937).

8 Scheibe, G., Kolloid. Z., 82, 1 (1938).

\section{Viscosity of Suspensions and the Einstein Equation}

THE Einstein equation $\eta=\eta_{0}(1+2 \cdot 5 \mathrm{~V})$ relating the viscosity $\eta$ of a suspension of solid spheres and $\eta_{0}$ the viscosity of the dispersion medium, $V$ being the volume in parts of a cubic centimetre occupied by the dispersed solid, has been used as a basis for determining the molecular structure of proteins and other colloidal substances ${ }^{1}$.

It is of interest in this connexion that the viscosity ratio $\eta / \eta_{0}$ is dependent, among other things, on temperature, the ratio being affected by the viscosity of the dispersion medium. The following data for (a) 16.7 per cent volume of graphite in a mineral lubricating oil and (b) 14.8 per cent volume kaolin in meta cresol, for example, illustrate this.

(a)

$\begin{array}{ccc}\text { Temp. }{ }^{\circ} \mathrm{C}, & \eta & \eta / \eta_{0} \\ 30 & 3 \cdot 7 & 3 \cdot 6 \\ 40 & 1 \cdot 9 & 3 \cdot 4 \\ 45 & 1 \cdot 5 & 3 \cdot 1\end{array}$

\begin{tabular}{|c|c|c|}
\hline (b) & Temp. ${ }^{\circ} \mathrm{C}$. & $\eta$ \\
\hline & $13 \cdot 5$ & $1 \cdot 21$ \\
\hline & 22 & $0 \cdot 15$ \\
\hline & 45 & 0.05 \\
\hline
\end{tabular}

$\eta / n_{0}$ $7 \cdot 7$ $7 \cdot 2$ $6 \cdot 7$

That the effect is not entirely due to disaggregation is indicated by using dispersion media of different viscosity. In the accompanying graphs, values of $\log \left(\eta / \eta_{0}-1\right)$ and $\log$ percentage volume are plotted for powdered silica in various liquids, and it will be seen that the curves are roughly in order of the viscosity of the liquids, the viscosity ratios being least for normal hexane and greatest with glycerol. The source of the variation is presumably due to the spinning motion of the particles or their aggregates,

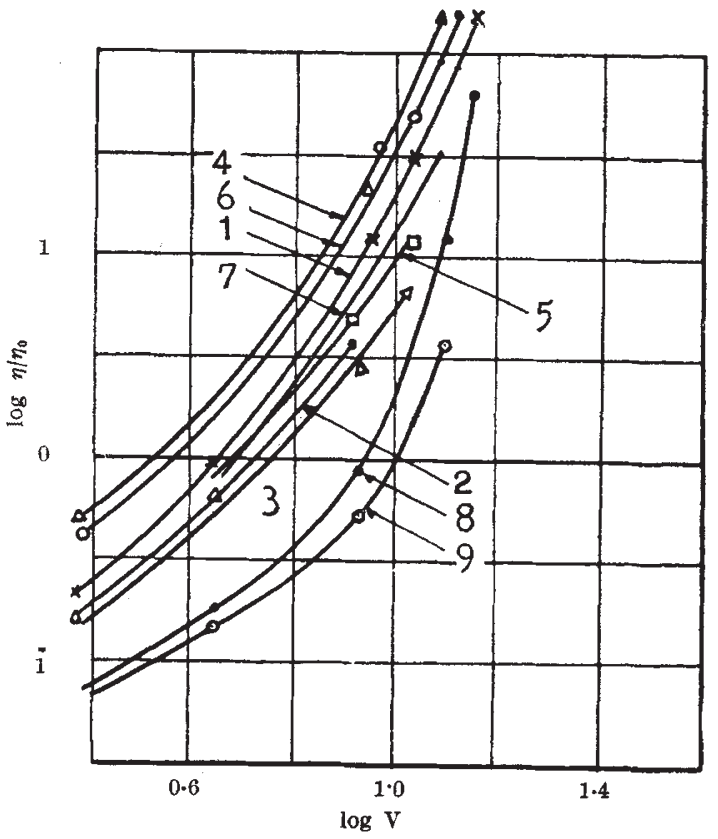

1, Mineral LUbricating OIL ; 2, Ricinoleic aCID 3, IIGHT MINERAL LUBRICATING OIL; 4, GLYCEROI $(91 \%) ; 5$, POPPY SEED OIL ; 6, RAW LINSEED OIL 7, TRIACETIN ; 8, CARBON TETRACHLORIDE ; 9, NORMAL HEXANE.

less time being required in the thinner liquids for the dispersed phase to take up positions of streamline flow.

It was found under isoviscometric conditions that the specific character of the dispersior medium affects the viscosity ratio; thus, for example, with kaolin, 5 per cent volume, a viscosity ratio of $2 \cdot 15$ was found in a light mineral oil, but in oleic acid the ratio was $1 \cdot 3$ and in benzyl alcohol $1 \cdot 45$.

It is possible that in very dilute suspension these effects may be minimized, but in the systems examined at lower concentrations the variations were observed.

The viscosity measurements were made in a simple form of plastometer, low rates of shear being avoided.

Chemistry Department, E. W. J. Mardles.

Royal Aircraft Establishment,

Farnborough, Hants. April 29.

1"Viscosity and Molecular Structure", by H. Mark and R. Simha, NATURE, 145, 571 (April 13, 1940).

\section{Cohesion of Liquids}

THE recent interesting letters of Profs. M. Born and R. Fürth, published in NATURE of May 11, on the strength of solids, lead me to think that experiments which I am now carrying out on the strength of liquids under hydrostatic tension may be of interest to physicists working in this field. Liquids, owing to their homogeneity and absence of rigidity, possess certain advantages over solids for the study of cohesion. In my view, measurements of the greatest tension which can be applied to liquids under various 